\title{
Anpassungsstrategien an Klimawandelfolgen für den Obstbau im Mittelmeerklima - Wird der Obstbau zum Klimaschützer?
}

\author{
Michael Blanke ${ }^{1}$
}

Eingegangen: 27. August 2019 / Angenommen: 24. September 2021 / Online publiziert: 29. Oktober 2021

(c) Der/die Autor(en) 2021

\section{Zusammenfassung}

Zur Abschlussveranstaltung des Adapt2Clima-EU-Projekts zu „Klimawandelfolgen für die Landwirtschaft im Mittelmeerraum und Adaptionsstrategien“ trafen sich fast 160 Klimaforscher aus über 30 Ländern am 24.-25. Juni 2019 in Heraklion - mit Klimamodellen und Risikoanalyse bzw. Vulnerabilität verschiedener Mittelmeerregionen. Bei den obstbaulichen Vorträgen stellte sich einmal die Witterung zur Blüte als besonders kritisch heraus, wobei zum einen kühl-nasse Witterung die Bestäubung durch ausbleibendes Fliegen der Honigbienen verhinderte und heiße Witterung die Pollen verklebten und zum anderen Unwetter mit Starkregenfällen im Laufe des Sommers, z. B. in Skopelos im August 2015, die Fruchtqualität bzw. die Ernte und auf Kreta im Frühling 2019 die Blüte bzw. den Fruchtansatz in Frage stellten. Als relativ klimaresilient erscheinen Gebirgsregionen wie Imathia, Pella und Pilion sowie Kreta und Zypern aufgrund der Kombination aus ausreichendem Kältereiz im Winter (Chilling), geringer Spätfrostgefahr, Zugang zu Wasser und weniger Hitzeschäden. Besonders betroffen von Klimawandelfolgen im Mittelmeerraum sind Wein, Tomate und Olive - in der Reihenfolge ihres Wasserbedarfs und ihrer Empfindlichkeit gegenüber Hitze und Trockenheit. Folgende Anpassungsstrategien wurden diskutiert: 1) Wasserauffang- und Rückhaltebecken; 2) Regelung der Wasserrechte; 3) Tröpfchenbewässerung, „deficit irrigation" oder auch Verzicht auf Bewässerung; 4) Sorten mit hohem Wasserausnutzungsgrad (WUE); 5) geringe Pflanzdichten/extensiver Anbau; 6) Senkung der Evapotranspiration, z. B. durch Beschattung mit Schattier- oder Hagelnetzen; 7) Verzicht auf Bodenbearbeitung; 8) Kurzhalten des Grasaufwuchses oder cover crop im Winter aufgrund von Wasserund Nährstoffkonkurrenz sowie Brandgefahr; 9) bei Oliven Einbringen von geschreddertem Schnittholz (statt Verbrennen) und 10) der Pressrückstände bzw. -kuchen sowie 11) im Weinbau Anbauverschiebung in nördliche Bergregionen. Ein Teil dieser Maßnahmen bedeutet einen Bruch mit althergebrachten Traditionen, da sowohl das Pflügen bzw. die Bodenbearbeitung als auch das Verbrennen von Schnittholz in Olivenhainen im Besitz der älteren Landbevölkerung in manchen Regionen Griechenlands nicht wegzudenken sind und aus einer Zeit stammen, als Schredder noch unbekannt waren. Die vielversprechenden Ansätze im Mittelmeerraum können auch ein Umdenken im Obstbau und den Beginn einer neuen Ära bedeuten, wenn neben der Erzeugung hochwertiger Früchte Klima- bzw. $\mathrm{CO}_{2}$-relevante Aspekte bei den Kultur- und Pflegemaßnahmen berücksichtigt und entlohnt werden.

Schlüsselwörter Olive (Olea europaea) $\cdot$ Wein (Vitis vinifera $\mathrm{L}) \cdot \mathrm{CO}_{2}$-Speicherung $\cdot$ Hagelnetze $\cdot$ Klimawandel . Solidarische Landwirtschaft · Trockenheit · Wasserausnutzungsgrad

Michael Blanke

mmblanke@uni-bonn.de
1 INRES-Gartenbauwissenschaft, Universität Bonn, Auf dem Hügel 6, 53121 Bonn, Deutschland 


\title{
Adaption Strategies to Climate Change for Fruit Cultivation in Mediterranean Climate - Will Fruit Cultivation Become a Climate Protector?
}

\begin{abstract}
The final meeting of the Adapt2Clima EU project attracted nearly 160 participants involved in climate change in Heraklion in June 2019. The meeting covered modelling, risk analysis and vulnerability of the Mediterranean regions with respect to agriculture: The weather at flowering was identified as one of the prevailing hot spots, when flower pollination is either hampered by cold-wet weather preventing honeybees from flying or pollen sticking together in hot weather and storms with heavy rainfall during the summer and fruit development like on Skopelos in August 2015 or in Imathia in 2017/8. Mountainous areas such as Imathia and Pella in Macedonia and Pilion in Northeast Greece were identified as potentially climate resilient areas for fruit growing in the future based on the following resilience criteria: a) sufficient chilling in winter, b) low risk of a late frost, c) access to water and d) heat resilience. Grapevine cultivation is most affected by climate change, followed by tomato and then olive-in order of their water requirement and sensitivity to heat and drought The following adaption strategies against climate change were suggested for growing fruit in this region:1) securing water reservoirs; 2) regulation of water rights; 3 ) implementing water saving measures such as drip irrigation, deficit irrigation, partial root zone drying or no irrigation; 4) varieties with efficient water use (WUE); 5) extensive low plant/tree density; 6) reduction in evapotranspiration e.g. by shading using nets; 7) zero tillage; 8) grassed alleyways or cover crops (during winter); 9) in olive, mulching with shredded tree pruning residues; and 10) use of mill waste in the orchard; and 11) in grape, vineyard translocation towards to North and/or upwards into colder hillsides. Some of these measures represent a break with regional fruit growing traditions, where ploughing fields and particularly alleyways of olive orchards and burning pruned wood were an established practice and date back to a time, when wood shredder were largely unknown or uncommon; the great efforts in the med towards a combined fruit production with $\mathrm{CO}_{2}$ sequestration, however, are promising.
\end{abstract}

Keywords Olive (Olea europaea) · Grape (Vitis vinifera L) · Adaption strategies · Carbon sequestration · Climate change $\cdot$ Drought $\cdot$ Heat $\cdot$ Land stewardship $\cdot$ Water use efficiency (WUE) $\cdot$ Zero tillage

\section{Einleitung}

Fast 160 Klimaforscher aus über 30 Ländern trafen sich - vor der Corona-Pandemie - vom 24.-25. Juni 2019 in Heraklion zur Abschlussveranstaltung des Adapt2Clima-EUProjekts. Wie interessant dieses Thema „Klimawandelfolgen für die Landwirtschaft im Mittelmeerraum und Adaptionsstrategien" ist, zeigte die hohe Zahl der Beiträge und Teilnehmer, die teilweise auch erst später zu dem Projekt dazugestoßen sind.

Besonders für Regionen wie Bosnien, Steiermark, Ungarn bis zu Saudi-Arabien, Thailand, Südkorea und Australien mit mittelmeerähnlichem Klimaregionen heute oder in Zukunft nach dem letzten IPCC-Report (IPCC 2014) ist das Thema interessant, um sich über mögliche Anpassungsstrategien zu informieren.

\section{Veränderungen im Mittelmeer}

Der Wasserspiegel im Mittelmeer steigt nach Prof. Elena Xoplaki von der Universität Giessen nach dem moderaten $\mathrm{RCP} 4,5$ (representative concentration pathway; $4,5 \mathrm{~W} / \mathrm{m}^{2}$ ) bis 2030 um $5-9 \mathrm{~cm}$ (2011 bis 2030) und nach RCP 8,5 bis 2100 möglicherweise bis zu $50-74 \mathrm{~cm}$ und die Oberflä- chentemperatur erhöht sich um $1,5^{\circ} \mathrm{C}$ im östlichen und bis 2,5 ${ }^{\circ} \mathrm{C}$ im westlichen Mittelmeer (Tab. 1).

\section{Kostenlose Wetterdaten für alle}

Carlos Buontempo stellte das englische ECWMF Copernicus Programm vor, das jedem Interessenten kostenlos Zugriff auf weltweit verfügbare Wetterdaten wie Wasseroberflächen-, Luft- oder Bodentemperatur, Niederschlag, Frost, Wind- oder Luftdruck ermöglicht.

\section{Wetterveränderungen - Starkregen als neues Gefahrenrisiko für den Obstbau}

Das Klima im Mittelmeerraum ist nach Professor J. Bindi von der Universität Florenz durch trockene heiße Sommer und eine Regenzeit von Oktober bis März gekennzeichnet, in der das Gros des Regens fällt. Nach Prof. J. Olesen von der Universität Aarhus (Dänemark) lassen die Wetterprojektionen für den Mittelmeerraum erwarten, dass der Jahresniederschlag um bis zu ca. $20 \%$ abnimmt und Starkregenereignisse zunehmen. Neu sind Unwetter mit Starkregenfällen im Laufe des Sommers, z. B. in Skopelos im August 2015 mit starker Erosion und Schäden an Infrastruktur wie Straßen 
Tab. 1 Veränderungen in den Klimamodellen bzw. -prognosen (Temperaturerhöhung bis 2100)

\begin{tabular}{lllll}
\hline $\begin{array}{l}\text { Grad der Klima-wan- } \\
\text { delfolgen }\end{array}$ & $\begin{array}{l}\text { Neuer Ansatz nach IPCC } \\
(2014)\end{array}$ & $\begin{array}{l}\text { Temperaturerhöhung (bis } \\
2100)\end{array}$ & $\begin{array}{l}\text { Alter IPCC-Ansatz mit } \\
\text { Szenarien }\end{array}$ & $\begin{array}{l}\text { Temperaturerhöhung (bis } \\
2100)\end{array}$ \\
\hline Dramatisch & RCP 8,5 & $3,7^{\circ} \mathrm{C}$ & ${\mathrm{A} 1 F^{\mathrm{a}}}^{\mathrm{a}}$ & $4,0^{\circ} \mathrm{C}$ \\
- & - & & $\mathrm{A}^{\mathrm{a}}$ & $3,4^{\circ} \mathrm{C}$ \\
- & RCP 6,0 & $\mathrm{~B} 2($ und A1T) & $2,4^{\circ} \mathrm{C}$ \\
- & RCP 4,5 & $\mathrm{~B}^{\mathrm{a}}$ & $1,8^{\circ} \mathrm{C}$ \\
Gering-mild & RCP 2,6 & $1,8^{\circ} \mathrm{C}$ & - & \\
\hline
\end{tabular}

$R C P$ representative concentration pathway

${ }^{a}$ Emissions-Szenarien des IPCC-Sonderberichtes über Emissions-Szenarien (IPCC 2000)

Tab. 2 Klimawandelfolgen für Oliven und Steinobst (Kirschen und Pfirsiche) im Mittelmeerraum

\begin{tabular}{lllll}
\hline Obstart & Anbauregion & Phänologisches Stadium & Schaden & Ursache \\
\hline Pflaume & Imathia & Blüte 2015 & Kein Fruchtansatz, fehlende & Regen, Temp. \\
Kirsche & Imathia & Blüte 2015 & Bestäubung, kein Bienenflug & unter 11 ${ }^{\circ}$ C, \\
Olive & Kreta & Blüte 2019 & & Unwetter, Sturm, \\
Pfirsich & Imathia & Vorernte 2017/8/9 & Fruchtfall & \\
(Trocken-) Pflaume & Skopelos & Ernte 2015 & Kein Trocknen \\
\hline
\end{tabular}

sowie auf Kreta im März-Mai 2019 das Wegschwemmen alter Steinbrücken wie der Keritibrücke bei Chania. Auf Skopelos traf der unerwartete sommerliche Starkregen die Pflaumentrocknung (Tab. 2) der „Prunes“ im Freien (Blanke 2015).

\section{Phänologie: Blüte bzw. Blühzeitraum stark betroffen und in Projektionen weitgehend unberücksichtigt}

Prof. J. Santos sowie Drs. Drogoudi et al. (2020) stellten durch den Klimawandel bzw. warmen Winter hervorgeru- fene Blühverfrühung bei Obst und Wein von 1-2 Wochen vor. Von einer um einen Monat früheren Ernte spät reifender Pfirsiche und von Problemen mit der Witterung zur Blüte berichtete Dr. P. Drogoudi von Demeter Naoussa; bei kalter Witterung $<11^{\circ} \mathrm{C}$ fliegen die Honigbienen nicht mehr zur Bestäubung der Obstanlagen aus und bei heißer Witterung verkleben die Pollen. In den letzten drei Jahren kam es bei Pfirsichen und Nektarinen in Naoussa/Imathia (Abb. 1) kurz vor der Ernte durch Unwetter mit Starkregen (100 mm in 3 Tagen) mit plötzlichem Temperaturabfall nach längerer Trocken- und Hitzephase zu Fruchtfall, Bronzing (Verbraunen der Fruchtschale) und bei Pfirsichen in Imathia und Pella (Pantelidis et al. 2021) (Tab. 2).
Abb. 1 Herkünfte des Steinobsts - anhand der Kartonagen aus relativ klimaresilienten Bergregionen Griechenlands (z. B. Naoussa/Imathia)

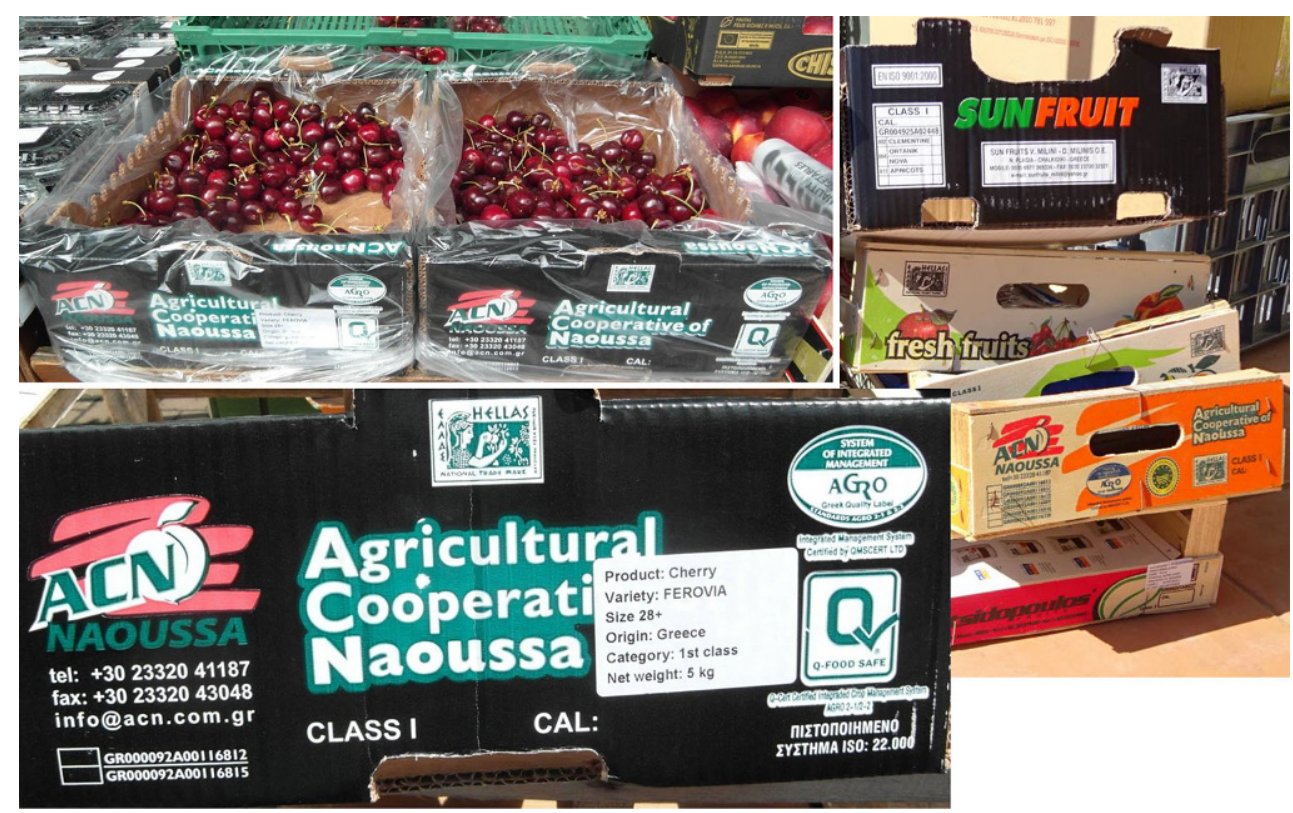


Tab. 3 Kriterien für klimaresilienten Obstbau (Drogoudi et al. 2020)

\begin{tabular}{ll}
\hline Witterung & Physiologie \\
\hline Ausreichend Chilling & Gute Bestäubung und Fruchtansatz \\
Geringe Spätfrostge- & Ausreichende Fruchtausfärbung, Frucht- \\
fahr & festigkeit und Fruchtgröße \\
Regen $>600 \mathrm{~mm} / \mathrm{Jahr}$ & Keine Hitzeschäden (Sonnenbrand) \\
\hline
\end{tabular}

\section{Klimaresiliente Regionen im Mittelmeerraum - trotz Klimawandel}

Ziel des gemeinsamen Beitrags aus Bonn und Mazedonien in Nordgriechenland war es, Kriterien für klimaresiliente Mittelmeerregionen zu entwickeln (Tab. 3) und solche Gebiete für den zukünftigen Obstbau zu identifizieren (Tab. 4). Während kleine Inseln ohne Hinterland besonders von den Klimawandelfolgen betroffen sind, kristallisieren sich umgekehrt für den Obstbau klimaresiliente Regionen heraus, in denen durch kulturtechnische Maßnahmen Arten- und Sortenwahl Abhilfe gegen Klimaveränderungen geschaffen werden kann. Dazu gehören Gebirgsregionen wie Imathia/ Pella in Mazedonien (Kirsch-, Pfirsich- und Weinbau) und Pilion (Apfelanbau), beide in Nordgriechenland mit Wäldern, gelegentlichem Schneefall im Winter und Skigebieten. Aber auch Inseln wie die Pflaumeninsel Skopelos (Blanke 2015), Kreta und Zypern zählen mit bis zu 1300 Chilling hours $(\mathrm{CH})$ bzw. $90 \mathrm{CP}$ (Tab. 4), d.h. für die meisten Obstarten ausreichende Kältestunden im Winter (Chilling) (Kaufmann et al. 2019), zu den klimaresilienten Zonen bis Juni mit frischem Quellwasser in den Bächen bis der erste Regen im Oktober fällt.

\section{Weinbau stärker vom Klimawandel betroffen als Oliven}

Besonders betroffen von Klimawandelfolgen im Mittelmeerraum sind Wein, Tomate und Olive - in der Reihenfolge ihres Wasserbedarfs und ihrer Empfindlichkeit gegenüber Hitze und Trockenheit. Der besonders betroffene Weinbau wird nach Prof. J. Olesen, Drs. M. Marikou und V. Sanchez in die Berge und weiter nördlich wandern, mit mehr alten Lokalsorten mit hoher Wasserausnutzung
(WUE) und klimaresilienten Rebsorten wie 'Syrah' ('Shi-

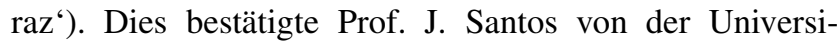
tät Porto (Portugal) mit erwarteten Ertragseinbußen im Weinbau in den südlichen Anbauregionen wie Portugal, Südspanien und Westtürkei, aber auch Ertragssteigerungen (um 5\%) in den Weinregionen in Frankreich, Deutschland, Ungarn, Norditalien (z.B. Südtirol) und den Balkanstaaten (Slovenien etc.).

\section{Bekannte Pflegemaßnahmen als Anpassungsstrategien - trotz Bruch mit althergebrachten obstbaulichen Traditionen}

$\mathrm{Zu}$ den in der Erprobung befindlichen Anpassungsstrategien gehören nicht nur Anbau alter regional an das Klima angepasster Sorten mit hoher Wasserausnutzungsfähigkeit (WUE), sondern auch der Bruch mit althergebrachten Traditionen: Sowohl das Pflügen bzw. Bodenbearbeitung als auch Verbrennen von Schnittholz in Olivenhainen sind in manchen Regionen Griechenlands bei der älteren Landbevölkerung nicht wegzudenken und stammen aus einer Zeit, als Schredder noch unbekannt waren (Abb. 2). Die in Tab. 5 aufgeführten Kultur- und Pflegemaßnahmen zielen insgesamt auf eine Verminderung der Wasserverluste (Abb. 3), d.h. weniger Evapotranspiration von Pflanze und Boden, Schattierung (Blanke 2007), Verzicht auf Bodenbearbeitung (zero tillage) und Kurzhalten des Aufwuchses. Frau Dr. G. Michalopoulos berichtete von neuen Anpassungsstrategien im Rahmen des EU-Projekts Oliveclima.eu: Kurz gehaltener Grasaufwuchs und zukünftiger Anbau ohne Bodenbearbeitung (Abb. 2) vermindern die Brandgefahr sowie Wasser- und Nährstoffkonkurrenz - ebenso das Schreddern von Schnittholz und Verwendung als Mulch und Einbringen der Pressrückstände aus der Olivenölgewinnung (Abb. 2).

\section{Rote Hagelnetze ganzjährig unter den Bäumen auf dem Boden}

In Hügellagen werden die Netze oft nicht zur Olivenernte, sondern das ganze Jahr über ausgespannt und ersetzen Mulchen und Bodenbearbeitung (Abb. 2) - rote (Hagel-)netze auf dem Boden, z.B. im Westen Kretas (Abb. 4) - sollen

Tab. 4 Kältereiz in relativ klimaresilienten Regionen Griechenlands (erste Schätzung der letzten drei Jahre; mit dem warmen Winter 2015/16, Drogoudi et al. 2017)

\begin{tabular}{lllll}
\hline Witterung & $\begin{array}{l}\text { Hügellagen } \\
\text { bis m }\end{array}$ & $\begin{array}{l}\text { Kältereiz in CH (Chilling } \\
\text { hours) }\end{array}$ & $\begin{array}{l}\text { Kältereiz in CU (Chilling } \\
\text { Units) }\end{array}$ & $\begin{array}{l}\text { Kältereiz in Chilling portions } \\
(\mathrm{CP})\end{array}$ \\
\hline Imathia, Pella, NO & $160-300 \mathrm{~m}$ & $944-1262 \mathrm{CH}$ & $1500-1941 \mathrm{CU}$ & $83-92 \mathrm{CP}$ \\
Pilion, Nordosten & $100-400 \mathrm{~m}$ & $\mathrm{Ca} .1350 \mathrm{CH}$ & $1600-1900 \mathrm{CU}$ & $\mathrm{Ca} .90 \mathrm{CP}$ \\
Heraklion, Kreta & $260 \mathrm{~m}$ & $762-1034 \mathrm{CH}$ & $1223-1658 \mathrm{CU}$ & $69-82 \mathrm{CP}$ \\
Zypern & $200-1000 \mathrm{~m}$ & $230-1360 \mathrm{CH}$ & $414-1940 \mathrm{CU}$ & $20-89 \mathrm{CP}$
\end{tabular}


Abb. 2 Verschiedene Anbausysteme am Beispiel Olivenanbau: Traditionelle Bodenbearbeitung bzw. Pflügen der Fahrgassen, das heute als durch verstärkten Humusabbau, $\mathrm{CO}_{2}$ Abgabe und Wasserverlust/ Evapotranspiration als negativ angesehen wird (a); Mulchen (b) zum Kurzhalten der Vegetation in der Fahrgasse (c) als klimaresilientes Anbauverfahren sowie Erosions- und Brandschutz, und Einbringen bzw. Rückführen des Presskuchen aus der Ölgewinnung unter die Bäume (d), was sowohl den Humusaufbaus als auch die carbon sequestration fördert, Wasserverluste vermindert und als klimaresilient und Klima förderlich angesehen wird
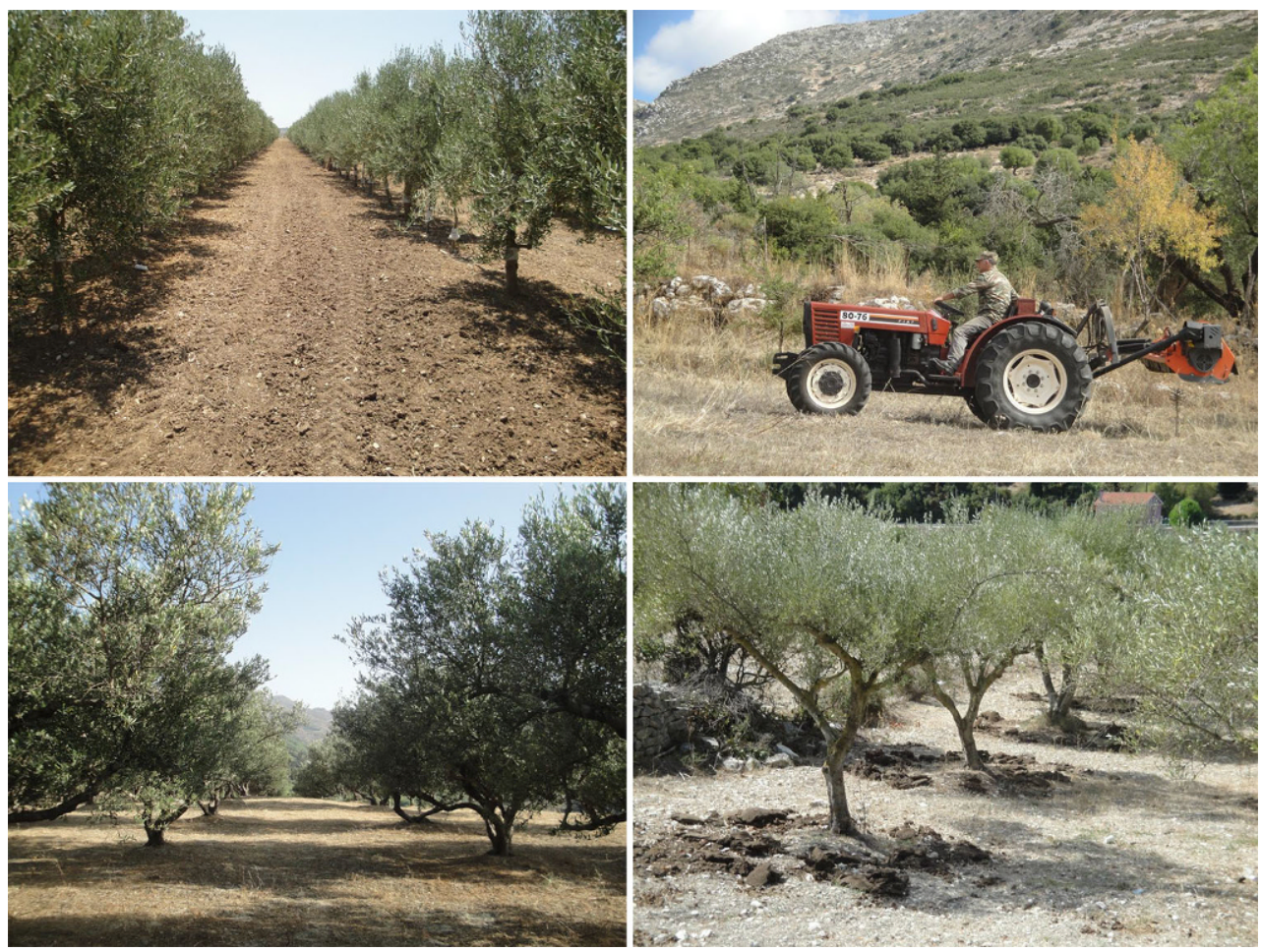

Tab. 5 Anpassungsstrategien an die Klimawandelfolgen im Mittelmeerraum

\begin{tabular}{ll}
\hline Klimawandelfolge & Anpassungsstrategien \\
\hline Hitze & Hitzeverträgliche Sorten ('Syrah') mit geringer WUE und Anbauverschiebung in nördliche Regionen (Weinbau) \\
Lange & 1) Wasserauffang- und Rückhaltebecken \\
Trockenphasen, & 2) Regelung der Wasserrechte \\
Wassermangel & 3) Tröpfchenbewässerung, deficit irrigation, patial rootzone drying oder auch Verzicht auf Bewässerung \\
(Absinken des & 4) Sorten mit hoher WUE \\
Grundwasserspie- & 5) Geringe Pflanzdichten/extensiver Anbau \\
gels; Versalzung) & 6) Senkung der Evapotranspiration, z. B. durch Schattier- oder Hagelnetze \\
& 7) Keine Bodenbearbeitung wie Pflügen \\
& 8) Kurzhalten des Grasaufwuchses oder cover crops im Winter durch Mulchen \\
& 9) Einbringen von geschreddertem Schnittholz (statt Verbrennen) und \\
10) der Pressrückstände bzw. -kuchen (Olive und Wein) \\
11) Anbauverschiebung in nördliche Regionen (Weinbau) \\
Keine Bodenbearbeitung und Mulchen als Erosionsschutz; Wasserauffangbecken \\
Auslichten dichter Waldbestände, extensiver Olivenanbau als Brandbarriere, Mulchen des Grasaufwuchs und Vermei- \\
den von Verbrennen von Schnittholz (Oliven)
\end{tabular}

${ }^{\mathrm{a}}$ Extensiver Olivenanbau entspricht ca. 500 Bäume/ha, intensiv ca. 2000 Bäume/ha

dabei die Photosynthese und den Ertrag erhöhen, was aber bisher nicht bewiesen ist.

\section{Obstbau als Kohlenstoffspeicher: von der $\mathrm{CO}_{2}$-Quelle (Source) zum $\mathrm{CO}_{2}$-Speicher (Sink) und Klimaschützer?}

Im Rahmen des EU-Projekts Oliveclima.eu (2019) wird versucht, die traditionelle Bodenbearbeitung mit Pflügen der Fahrgassen mit hohem $\mathrm{CO}_{2}$-Verlust (Bodenatmung) nicht nur in Klimaneutralität, sondern in das Gegenteil umzuwandeln: $\mathrm{CO}_{2}$-Speicher durch Einbringen von Pressrückständen aus der Olivenölgewinnung und Schreddern und Rückführen von Schnittholz, um den Humusgehalt zu erhöhen. Eigene Messungen in Bonn hatten gezeigt, dass die $\mathrm{CO}_{2}$-Abgabe des frei gehaltenen Baumstreifens (Bodenatmung) im gemäßigten Klima bei unseren fruchtbaren Böden die $\mathrm{CO}_{2}$-Aufnahme der Apfelbäume - über das Jahr berechnet - erreichen oder sogar übersteigen kann (Blanke 
Abb. 3 Verschiedene Bewässerungsformen am Beispiel Olivenanbau: Beregnungsschlauch auf dem Boden (a); Beregnungsschlauch im Baum, um mechanische Bodenbearbeitung zu möglichen - auch zur Evaporationskühlung (b); Bewässerungsbecken (c) und Wasserverteiler (d)

Abb. 4 In Hügellagen bleiben die Netze oft nicht nur zur Olivenernte, sondern das ganze Jahr über aufgespannt und ersetzen Mulchen und Bodenbearbeitung. Rote (Hagel-)netze auf dem Boden $(\mathbf{a}, \mathbf{b})$ - hier auf Kreta sollen die Photosynthese und den Ertrag erhöhen, was noch zu beweisen bleibt
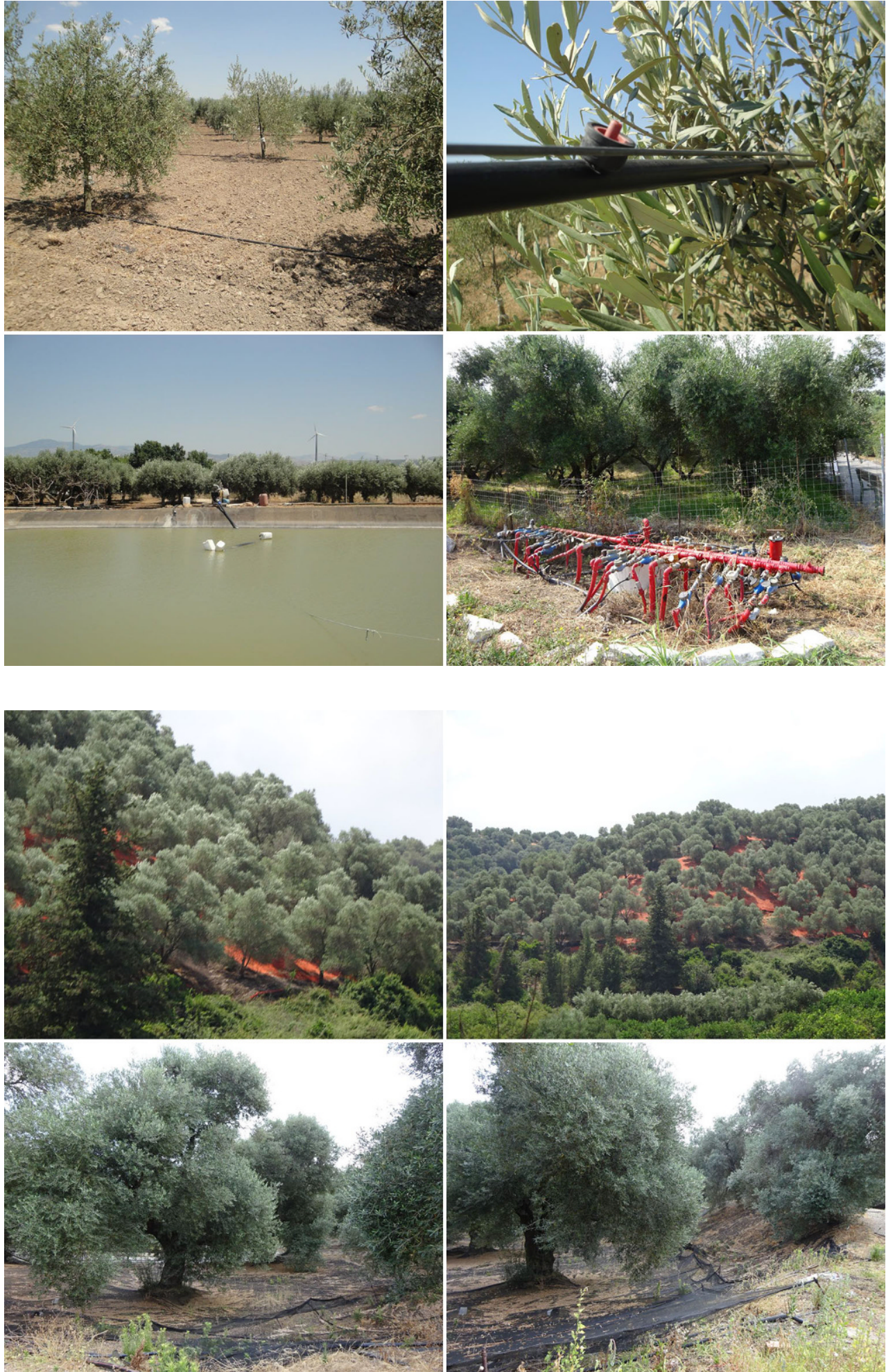
Abb. 5 Traditionelle Eddy-Covariance-Messungen der $\mathrm{CO}_{2}$ Flüsse in einer Apfelanlage im Trentino von V. Zanotelli und M. Tagliavini sowie die neu vorgestellte gleichzeitige Messung der drei klimaschädlichen Gase $\mathrm{CO}_{2}, \mathrm{CH}_{3}$ und $\mathrm{N}_{2} \mathrm{O}$ (@) Maria Doula) mittels LIDAR (b)

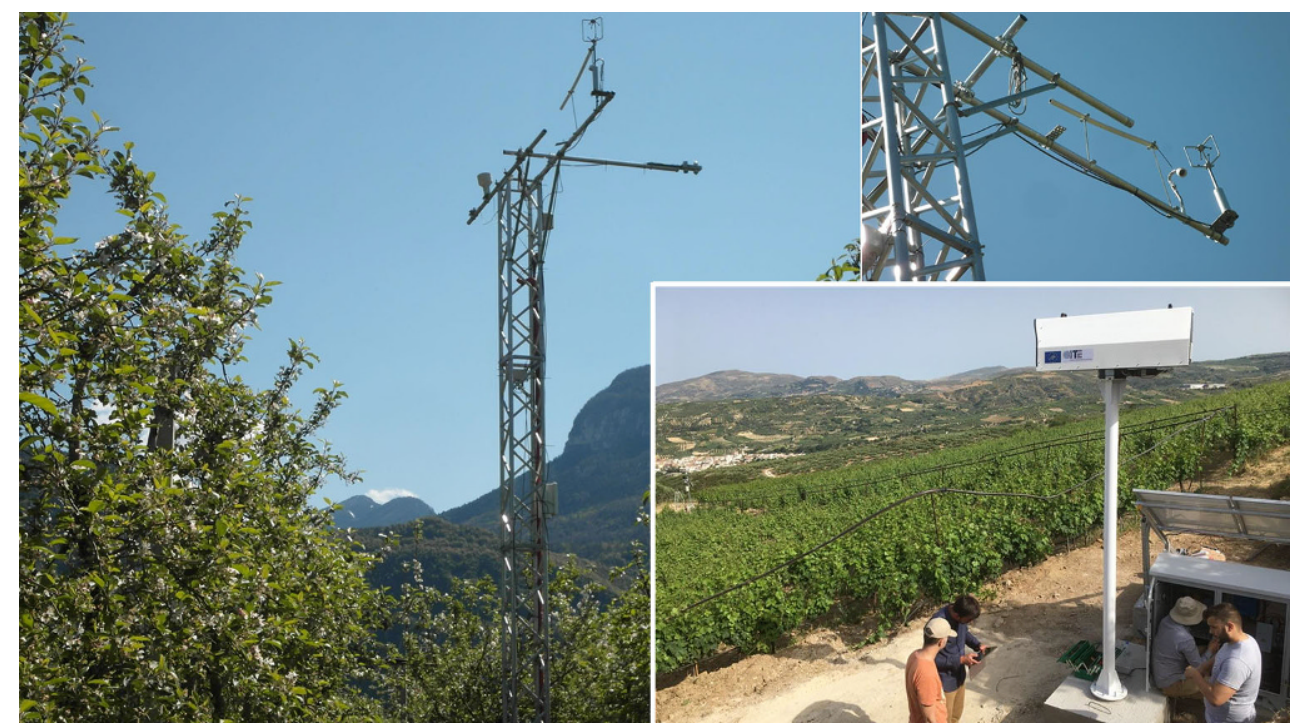

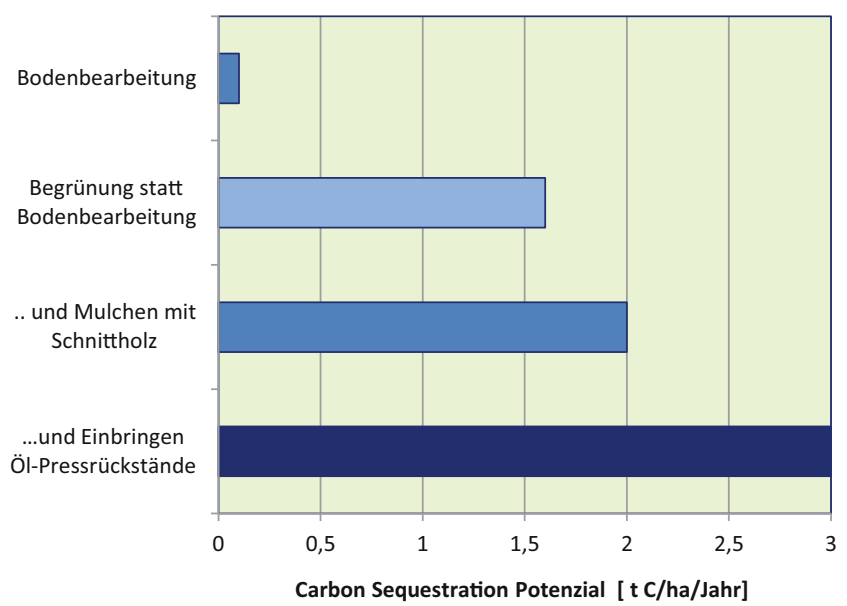

Abb. 6 Schematisierung des $\mathrm{CO}_{2}$-Speicher-(carbon sequestration) Potenzials am Beispiel Oliven in Abhängigkeit von den Pflege- und Kulturmaßnahmen. Zusammenstellung aus der Literatur; damit kann aus der Obstanlage unter bestimmten Bedingungen ein $\mathrm{CO}_{2}$-Speicher werden
1997); Messungen von V. Zanotelli und M. Tagliavini an ganzen Apfelbaumbeständen im Trentino (Abb. 5) wiesen auf die Möglichkeit der carbon sequestration hin (Scandellari et al. 2016). Olivenhaine weisen aufgrund ihrer Langlebigkeit bei Begrünung der Fahrgassen, Verzicht auf Bodenbearbeitung und Zufuhr organischer Substanz wie Pressrückständen ein relativ hohes Potenzial auf von bis zu 5-7t C/ha/Jahr Kohlenstoffspeicherung (carbon sequestration) (Tab. 6). Dabei sind die $\mathrm{CO}_{2}$-Speicherraten nach Umstellung der Kulturmaßnamen am Anfang am höchsten und nehmen dann schnell ab. Im Olivenanbau fallen 0,4-1,0t TM Schnittholz an, wobei letztere bei Verwendung als Mulch zu Beginn der Maßnahme anfangs bis $\mathrm{zu} 0,55 \mathrm{t} \mathrm{C} / \mathrm{ha} / \mathrm{Jahr}$ Kohlenstoff speichern kann (carbon sequestration). Manche Olivenbäume werden jährlich, andere nur in Alternanzjahren und manche alle 3-6 Jahre zu unterschiedlichen Zeiten (Winter, Frühling oder Sommer) geschnitten; dies wurde bei der Auswahl der 10 Pilotfarmen in Griechenland im Projekt berücksichtigt. Voraussetzung für carbon sequestration von Olivenhainen sind daher a) Begrünung, b) keine Bodenbearbeitung (zero tillage), c) Rückführung des Schnittholzes und d) der Pressrück-

Tab. 6 Literaturübersicht über Kohlenstoffspeicherung (carbon sequestration) in Obstanlagen mit Mittelmeerklima (eigene Zusammenstellung)

\begin{tabular}{llll}
\hline Obstart & C-Speicher & Lage/Klima & Quelle \\
\hline Olivenhain & $7,5 \mathrm{t} \mathrm{C} / \mathrm{ha} / \mathrm{Jahr}$ & Mittelmeerraum-Italien (Bologna, Materra, Sizilien) & Scandellari et al. (2016) \\
Apfel & $4,3 \mathrm{t} \mathrm{C/ha/Jahr}$ & & \\
Wein & $2-7 \mathrm{t} \mathrm{C} / \mathrm{ha} / \mathrm{Jahr}$ & & \\
Wein & $0,05 \mathrm{t} \mathrm{C} / \mathrm{ha} / \mathrm{Jahr}$ & Maremma, Toskana & Bosco et al. (2011) \\
Wein & $0,78 \mathrm{t} \mathrm{C} / \mathrm{ha} / \mathrm{Jahr}$ & $\ldots$ mit begrünter Fahrgasse & Vicente-Vicente et al. (2016) \\
Mandel & $2,0 \mathrm{t} \mathrm{C} / \mathrm{ha} / \mathrm{Jahr}$ & & \\
Olivenhain & $1,1 \mathrm{t} \mathrm{C} / \mathrm{ha} / \mathrm{Jahr}$ & & \\
Olivenhain & $5,3 \mathrm{t} \mathrm{C} / \mathrm{ha} / \mathrm{Jahr}$ & $\ldots$ und zusätzlich mit Pressrückständen & \\
\hline
\end{tabular}


stände bei der Olivenölgewinnung (Abb. 6) - Ansätze, die den carbon footprint der Kulturen vermindern könnten.

\section{Wiederbewirtschaftung brach liegender Terrassen und land stewardship-Projekte}

Durch die wirtschaftliche Krise Griechenlands der letzten Jahre (2010-2018) ist ein Teil der Stadtbevölkerung in ihre ländlichen Ursprungsorte zurückgekehrt und bestellt nicht nur brachliegende Felder, sondern ist nach Frau Dr. Mylona auch bei der Wiederaufforstung brachliegender Terrassen - bekannt vom Weinbau in Steillagen an Rhein und Mosel beteiligt und auf der Insel Andros vor Athen zu finden. Dabei finden z.T. neue Konzepte Anklang. Die solidarische Landwirtschaft (2019) (SoLaWi, land stewardship) beteiligt die Inselbewohner sowohl an den Kosten als auch der Feldarbeit und hilft, sich einen Ernteanteil zu sichern, die Landschaft zu erhalten, Erosion verhindern und zur $\mathrm{CO}_{2-}$ Bindung statt Bodenatmung beizutragen.

\section{Waldbrand}

Weinbau oder extensive Olivenhaine können als Brandschneisen zwischen den Kiefernwäldern fungieren. Das Vermeiden von Verbrennen von Schnittholz, Kurzhalten des Grasaufwuchses und das Entfernen von unerwünschtem Aufwuchs (Auslichten von zu dichten jungen Waldbeständen) kann dazu beitragen, das Waldbrandrisiko zu senken, seiner Ausbreitung entgegenzuwirken und seine Bekämpfung zu erleichtern (Tab. 5) - unklar blieb in dem Vortrag von Frau Dr. K. Radoglou, warum diese Auslichtung mit erhöhten Lachgasemissionen verbunden war.

\section{Neue effektive Veranstaltungs- und Veröffentlichungsform}

Im Gegensatz zu den gartenbaulichen Tagungen war die Adapt2Clima-Tagung Teil einer einwöchigen Präsenz-Veranstaltungsreihe, die mit Water and Waste Management mit demselben Sekretariat, welcome desk, Posterwänden in denselben Räumlichkeiten und dann circular economy mit Exkursion nach Rethymo fortgesetzt wurde und so den organisatorischen Aufwand für den Auf- und Abbau effektiv gestaltete.

Statt der Proceedings als Actaband, der bei der SHE (Symposium on Horticulture in Europe) 2008 in Angers, beim cherry congress 2014 und SHE 2016 in Chania drei Jahre in Anspruch nahm, verhandelten die Organisatoren mit referierten Journalen. Die besten Beiträge der Adapt2Clima wurden ausgewählt und bereits zeitnah in
„Water and Waste Management“ oder im „EU-Mediterranen Journal for Integrating Environment" als Sammelband "Climate change impacts and adaptation options with emphasis on the agricultural sector in the Mediterranean basin" bei Springer als Sonderheft veröffentlicht.

\section{Fazit}

Insgesamt wurden auf der Adapt2Clima viele Anpassungsstrategien an die Klimawandelfolgen für den Obst- und Weinbau vorgestellt. Diese Ideen und teilweise neuen Konzepte brechen aber auch z.T. mit althergebrachten obstbaulichen Traditionen. Für die Umsetzung dieser Konzepte war die auf der Abschlusstagung des Adapt2Clima praktizierte Kombination mit dem $\mathrm{CO}_{2}$-Fußabdruck landwirtschaftlicher Kulturen im Mittelmeerraum sowie die Zusammenarbeit mit den örtlichen Gemeinden (Municipality) und staatl. Wasser- und Stadtplanungsbehörden ein wertvoller und Erfolg versprechender Ansatz.

Danksagung Der Dank gilt Konstantinos Moustakas für perfekte Vorbereitung mehrerer Tagungen gleichzeitig. Durch seinen unermüdlichen Einsatz und die flexible Programmgestaltung wurde die Adapt2Clima zur perfekt vorbereiteten Tagung Griechenlands.

Funding Open Access funding enabled and organized by Projekt DEAL.

\section{Einhaltung ethischer Richtlinien}

Interessenkonflikt M. Blanke gibt an, dass kein Interessenkonflikt besteht.

Ethische Standards Für diesen Beitrag wurden von den Autoren keine Studien an Menschen oder Tieren durchgeführt. Für die aufgeführten Studien gelten die jeweils dort angegebenen ethischen Richtlinien.

Open Access Dieser Artikel wird unter der Creative Commons Namensnennung 4.0 International Lizenz veröffentlicht, welche die Nutzung, Vervielfältigung, Bearbeitung, Verbreitung und Wiedergabe in jeglichem Medium und Format erlaubt, sofern Sie den/die ursprünglichen Autor(en) und die Quelle ordnungsgemäß nennen, einen Link zur Creative Commons Lizenz beifügen und angeben, ob Änderungen vorgenommen wurden.

Die in diesem Artikel enthaltenen Bilder und sonstiges Drittmaterial unterliegen ebenfalls der genannten Creative Commons Lizenz, sofern sich aus der Abbildungslegende nichts anderes ergibt. Sofern das betreffende Material nicht unter der genannten Creative Commons Lizenz steht und die betreffende Handlung nicht nach gesetzlichen Vorschriften erlaubt ist, ist für die oben aufgeführten Weiterverwendungen des Materials die Einwilligung des jeweiligen Rechteinhabers einzuholen.

Weitere Details zur Lizenz entnehmen Sie bitte der Lizenzinformation auf http://creativecommons.org/licenses/by/4.0/deed.de. 


\section{Literatur}

Blanke MM (2015) Agioritiko - lukrativer Trockenpflaumenanbau in Griechenland. Obstbau 2015(12):707-710

Blanke MM (2007) Farbige Hagelnetze: Ihre Netzstruktur sowie Licht- und UV-Durchlässigkeit bestimmen die Ausfärbung der Apfelfrüchte. Erwerbs-Obstbau 49(4):127-139. https://doi.org/ 10.1007/s10341-007-0048-6

Blanke MM (1997) $\mathrm{CO}_{2}$ fluxes in an apple orchard. In: Mohren G et al (Hrsg) Impacts of global change on tree physiology and forest ecosystems. Nov. 1996, Bd. 52. Kluwer Academic Publishers, Wageningen, S 173-186

Bosco S, Di Bene C, Galli M, Remorini D, Massai R, Bonari E (2011) Greenhouse gas emissions in the agricultural phase of wine production in the Maremma rural district in Tuscany, Italy. Italian J Agron 6(2):e15

Drogoudi P, Kazantzis C, Blanke MM (2017) Effects of climate change on cherry flowering in Macedonia, Northern Greece. Proc. COST Cherry FA 1104 Action Working Group 2 'Cherry phenology and climate change' Workshop Ullensvang, April 2014, (Blanke M M et al., Hrsg.). Acta Hortic 1162:45-53. https://doi.org/10.17660/ ActaHortic.2017.1162.8

Drogoudi P, Kazantzis K, Kunz A, Blanke MM (2020) Effects of climate change on cherry production in Naoussa, Greece and Bonn, Germany: adaptation strategies. Euro Mediterranean J Environ Integr. https://doi.org/10.1007/s41207-020-0146-5

IPCC (2000) Nebojsa Nakicenovic and Rob Swart (Eds.) Cambridge University Press, UK. pp 570. https://www.ipcc.ch/report/ emissions-scenarios/. Zugegriffen: 2. Juni 2019
IPCC (2014) Climate change 2014: mitigation of climate change. Contribution of working group III to the fifth assessment report of the intergovernmental panel on climate change. IPCC, Cambridge, New York

Kaufmann H, Kunz A, Blanke MM (2019) Chilling requirements of Mediterranean fruit crops in a changing climate. Acta Hortic 1242:275-280. https://doi.org/10.17660/ActaHortic.2019.1242. 38

Oliveclima.eu (2019) https://www.oliveoiltimes.com/olive-oil-basics/ project-greece-converts-olive-cultivation-climate-managementtool/54934. Zugegriffen: 10. Juli 2019

Pantelidis G, Mavronlatis T, Drogoudi P (2021) Consecutive wet days may impede fruit quality of peach and nectarine and cause fruit drop. Sci Hortic. https://doi.org/10.1016/j.scienta.2021.110011

Scandellari F, Caruso G, Liguori G, Meggio F, Palese AM, Zanotelli D, Celano G, Gucci R, Inglese P, Pitacco A, Tagliavini M (2016) A survey of carbon sequestration potential of orchards and vineyards in Italy. Eur J Hortic Sci 81(2):106-114. https://doi.org/10.17660/ eJHS.2016/81.2.4

Solidarische Landwirtschaft https://www.solidarische-landwirtschaft. org. Zugegriffen: 20. Aug. 2019

Vicente-Vicente JL, García-Ruiza R, Francavigliab R, Aguilerac E, Smith P (2016) Soil carbon sequestration rates under Mediterranean woody crops using recommended management practices: a meta-analysis. Agric Ecosys Environ 235:204-214. https://doi. org/10.1016/j.agee.2016.10.024 